12

\title{
Моделирование фазовых сдвигов света в порядках дифракционных решеток интерференционного датчика линейных перемещений
}

\author{
(C) С.Б. Одиноков ${ }^{1}$, М.В. Шишова ${ }^{1, \uparrow}$, А.Ю. Жердев ${ }^{1}$, М.С. Ковалев ${ }^{1}$, М.Л. Галкин ${ }^{1}$, В.Ю. Венедиктов ${ }^{2,3}$ \\ ${ }^{1}$ МГТУ им. Н.Э. Баумана, \\ 105005 Москва, Россия \\ ${ }^{2}$ Санкт-Петербургский государственный электротехнический университет „ЛЭТИ“ им. В.И. Ульянова, \\ 197376 Санкт-Петербург, Россия \\ ${ }^{3}$ Санкт-Петербургский государственный университет, \\ 199034 Санкт-Петербург, Россия \\ ฯ e-mail: mshishova@bmstu.ru
}

Поступила в редакцию 20.03.2019 г.

В окончательной редакции 11.04.2019 г.

Принята к публикации 23.04.2019 г.

\begin{abstract}
Рассмотрены фазовые сдвиги света в порядках дифракционных решеток для интерференционного датчика линейных перемещений. Применение фазовой дифракционной решетки с заданными геометрическими параметрами поверхностного рельефа позволяет стабилизировать фазовые соотношения в оптических сигналах и в результате в итоговых сигналах, снимаемых с датчика перемещений. На основе данных математического моделирования предложены технически реализуемые параметры поверхностного рельефа дифракционных решеток для создания требуемых фазовых сдвигов между дифрагированными пучками и сигналами квадратурной модуляции и для достижения требуемой точности измерений с нанометрическим разрешением.
\end{abstract}

Ключевые слова: фазовый сдвиг, датчик линейных перемещений, квадратурные сигналы.

DOI: $10.21883 /$ OS.2019.09.48205.112-19

\section{Введение}

На сегодняшний день измерения линейных и угловых перемещений с нанометрическим разрешением обеспечиваются интерференционными оптическими датчиками [1-4]. В таких датчиках при равномерном линейном перемещении измерительной головки относительно кодирующей шкалы формируются гармонические сигналы, последующее декодирование которых в электронном блоке позволяет получить величину перемещения с погрешностью единиц нанометров. Оптические сигналы, характеризующие линейные перемещения, формируются в оптической системе датчика и сохраняют постоянный период интерференционной картины в пространстве с помощью рельефно-фазовых дифракционных решетокизмерительных шкал. Для обеспечения высокого разрешения датчика, а также для определения направления линейного перемещения необходимы как минимум два оптических сигнала, составляющие квадратурную пару: синусоидальный SIN и косинусоидальный $\operatorname{COS}[5,6]$. Период оптических сигналов пропорционален периоду измерительной шкалы. Шаг измерения формируется путем дискретизации уже электрических сигналов в электронном блоке и вычисления обратной гармонической функции. Тогда величина линейного перемещения внутри периода гармонического сигнала будет определяться фазой текущего уровня оптического сигнала. Причем стабильность разности фаз между оптическими сигналами в процессе перемещения определяет погреш- ность дискретизации внутри периода сигнала, а значит, величины разрешения и погрешности измерения самого датчика.

Традиционно в оптических системах интерференционных датчиков линейных перемещений стабилизация разности фаз оптических сигналов достигается либо добавлением новых фазовых оптических элементов, либо различными юстировками $[5,7]$ оптико-механических элементов датчика, например, сдвигом-поворотом дифракционной решетки в измерительной головке, наклоном лазера, перекрытием части апертуры пучка и др. Проблема данного подхода выражается в следующем недостатке. В процессе оптико-механических юстировок, движения привода и перемещения измерительной головки относительно кодирующей шкалы (КШ) вследствие несовершенства каретки привода, погрешностей направляющих и др. происходит нарушение параллельности измерительной кодирующей шкалы и измерительной головки с анализирующей шкалой (АШ). Это приводит к дестабилизации фазовых сдвигов в оптических сигналах из-за изменения длины пути и к внесению дополнительной погрешности при измерениях. Использование пропускающей дифракционной решетки с заранее сформированными фазовыми сдвигами в рабочих дифракционных порядках в качестве элемента фазовой задержки позволит обеспечить в оптической схеме датчика стабильную квадратурную модуляцию оптических сигналов без проведения сложной дополнительной оптико-механической или оптической юстировки. 


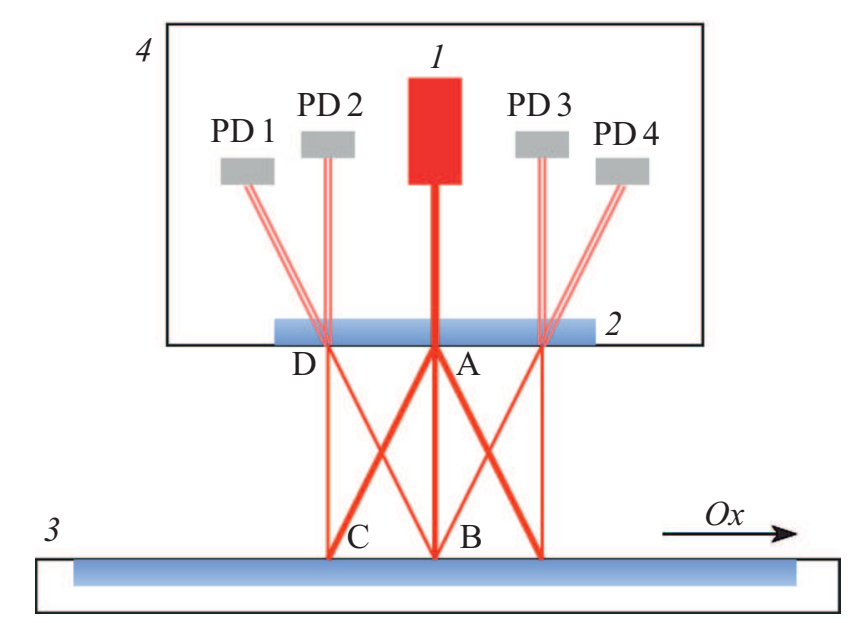

Рис. 1. Оптическая схема интерференционного датчика линейных перемещений: 1 - лазерный модуль, 2 - анализирующая шкала, 3 - кодирующая шкала, 4 - измерительная головка.

Настоящая работа посвящена моделированию фазовых сдвигов в порядках дифракционных решеток и расчету параметров их поверхностного рельефа, обеспечивающих повышение точности измерения перемещений до единиц нанометров, выполняемых с помощью интерференционного датчика линейных перемещений.

\section{Принцип работы интерференционного датчика линейных перемещений}

Принцип измерения линейных перемещений в интерференционных датчиках основан на внесении стабильно постоянного фазового сдвига в оптические сигналы [5-7], формируемые за счет интерференции световых пучков на фотоприемниках. На рис. 1 приведена оптическая схема интерференционного датчика линейных перемещений на основе дифракционных решеток. Применение высокочастотных дифракционных решеток с одинаковыми периодами, сравнимыми с длиной волны источника излучения, в качестве кодирующей шкалы, которая линейно перемещается относительно измерительной головки, и в качестве анализирующей шкалы, расположенной внутри измерительной головки, позволяет значительно повысить точность измерений такого датчика [8]. При этом АШ представляет собой пропускающую фазовую дифракционную решетку, а КШ представляет собой отражательную фазовую дифракционную решетку, каждая из которых выполняет функции как делителя пучка лазерного излучения, так и оптического элемента для внесения фазового сдвига в дифрагированные пучки света. После дифракции лазерного излучения на этих двух АШ и КШ пучки света в определенных порядках дифракции попарно складываются и интерферируют на фотоприемниках ФП1-ФП4 (photodetectors PD1-PD4) в измерительной головке, образуя „бесконечные“ интерференционные полосы. Смещение дифракционных решеток друг относительно друга приводит к изменению фазы в дифракционных порядках и к изменению структуры интерференционных полос (в случае линейного перемещения - к их сдвигу) в плоскости фотоприемников и, таким образом, к изменению амплитуд итоговых электрических сигналов, снимаемых с фотоприемников. В результате интерференции формируются оптические сигналы с попарной разностью фаз, равной $90^{\circ}$, например, между фотоприемниками ФП1 и ФП2 и симметрично между ФП3 и ФП4. Таким образом, оптически обеспечивается квадратурная модуляция оптических сигналов, а распределение интенсивности света в плоскости каждого из четырех фотоприемников ФП1-ФП4 представляет собой гармоническую функцию от величины линейного сдвига объекта вдоль координаты $x$. При этом главным является требование к стабильности первоначальных фазовых сдвигов (фазовых задержек) между квадратурными оптическими сигналами в плоскости фотоприемников.

Рассмотрим более подробно ход лучей в оптической схеме датчика и формирование интерференционных оптических сигналов при линейных перемещениях шкал (рис. 2). Сплошными линиями изображены пучки света, используемые для формирования оптических сигналов, а штриховой линией обозначены пучки света, не используемые в оптической схеме и в которых дифракционная эффективность минимизируется. В оптической схеме на рис. 2, a показан ход лазерных пучков, дифрагировавших на АШ (пропускающей дифракционной решетки) и до КШ (отражательной дифракционной решетки), а также дифрагировавших на ней пучков в обратном ходе. В оптической схеме на рис. $2, b$ показан обратный ход лучей при распространении дифрагировавших пучков, отраженных от КШ и до АШ. Причем рассматриваются только пучки в \pm 1 -х дифракционных порядках, а пучки лазерного излучения более высоких дифракционных порядков не попадают в оптическую схему датчика и в дальнейшем моделировании не рассматриваются.

Рисунок 2, $a$ иллюстрирует внесение фазового сдвига при первой дифракции на анализирующей шкале через пропускающую дифракционную решетку, представляющую собой анализирующую шкалу, распространяется в трех направлениях, соответствующих +1-м, 0 и -1-м порядкам дифракции. АШ должна быть сконструирована таким образом, чтобы прошедший луч (отрезок $\mathrm{AB})$ опережал дифрагировавший (отрезок АC) по фазе. Обозначим эту величину фазового сдвига $\Phi_{1}$. Далее излучение дифрагирует на отражательной дифракционной решетке, выполняющей роль кодирующей шкалы в датчике. КШ подвижна, и вносит фазу $2 \pi$ при ее перемещении на величину в один период $d$. Тогда при перемещении на величину х вносимая в дифракционный порядок фаза света равна $\Omega= \pm 2 \pi x / d$. Причем данный сдвиг фазы прибавляется к текущему при дифракции в +1 -й порядок $(+\Omega)$ и вычитается при дифракции в -1 -й порядок $(-\Omega)$. После дифракции на КШ каждый пучок 


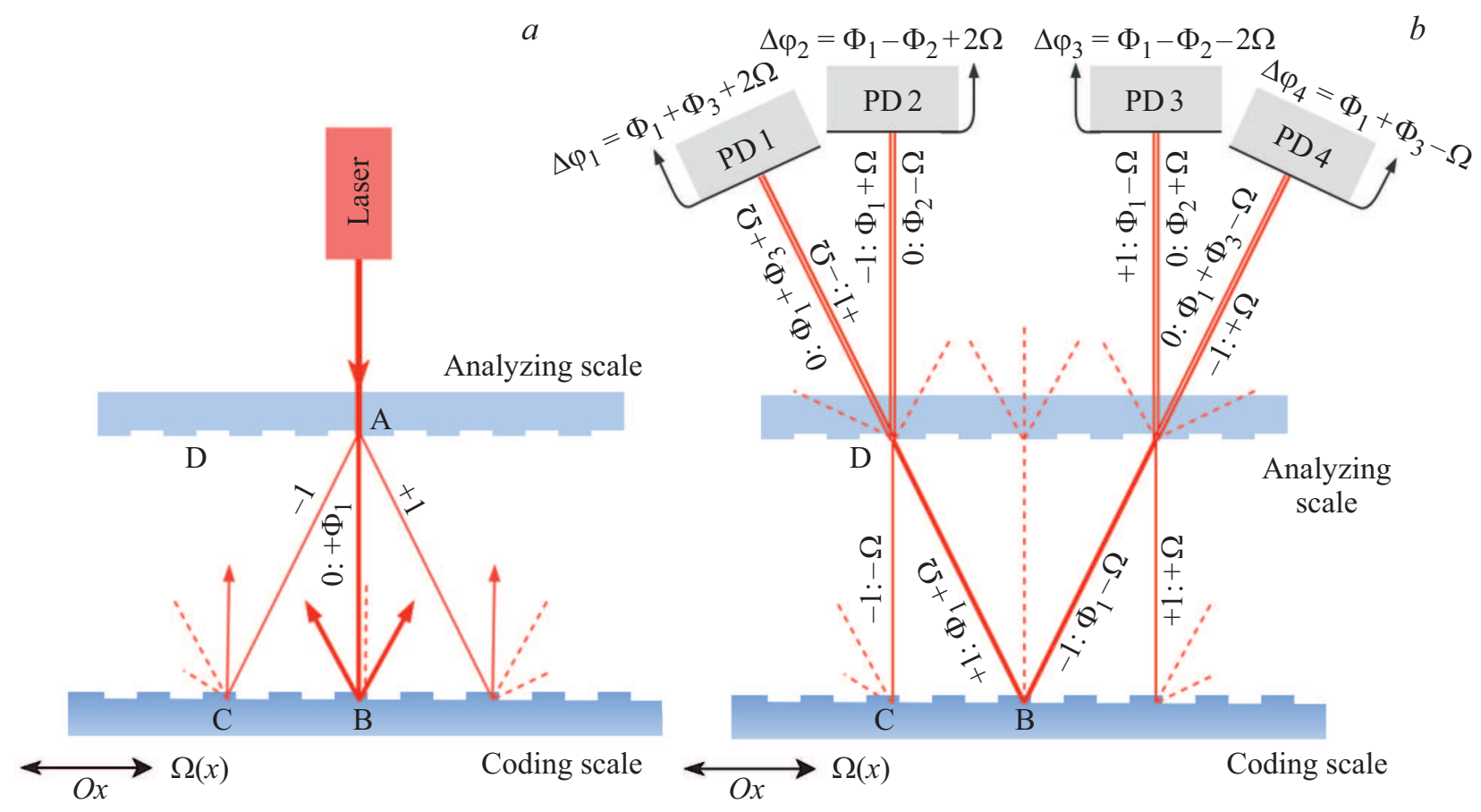

Рис. 2. Ход лучей в оптической схеме: $(a)$ - при распространении излучения от источника до кодирующей шкалы, $(b)-$ при распространении после дифракции на кодирующей шкале.

снова попадает на АШ, на которой опять дифрагирует в обратном ходе. Прошедшие пучки снова приобретают некоторый фазовый сдвиг относительно дифрагировавших пучков, как показано на рис. $2, b$. При нормальном падении излучения со стороны АШ обозначим величину фазового сдвига $\Phi_{2}$, а при наклонном падении как $\Phi_{3}$. Пары пучков, которые распространяются в одном направлении, интерферируют в плоскости каждого ФП1-ФП4.

В предложенной оптической схеме в силу симметрии обеспечивается равенство оптических длин путей интерферирующих пучков. Прошедшие пучки снова приобретают некоторый сдвиг фаз относительно дифрагировавших. При нормальном падении излучения со стороны решетки в направлении $\mathrm{CD}$ в 0-м порядке относительно +1-го порядка формируется фазовый сдвиг $\Phi_{2}$. При наклонном падении излучения в направлении $\mathrm{BD}$ величина фазового сдвига в 0-м порядке относительно +1 -го порядка составляет $\Phi_{3}$. Пары пучков, которые распространяются в одном направлении, интерферируют в плоскости каждого ФП1-ФП4. В предложенной оптической схеме в силу симметрии обеспечивается равенство оптических путей интерферирующих пучков,

Если оптическая схема сьюстирована, на каждом из приемников наблюдается интерференционная полоса бесконечной ширины. Интенсивность света каждого из интерференционных оптических сигналов, полученных при интерференции пучков с комплексными амплитудами световых волн $A=|A| \exp \left(i \Phi_{\mathrm{A}}\right)$ и $B=|B| \exp \left(i \Phi_{\mathrm{B}}\right)$ в плоскости каждого $k$-го фотоприемника (от ФП1 до ФП4), определяется по формуле

$$
\begin{aligned}
I_{k} & =\left|A_{k}\right|^{2}+\left|B_{k}\right|^{2}+2\left|A_{k}\right|\left|B_{k}\right| \cos \left(\Phi_{\mathrm{A} k}-\Phi_{\mathrm{B} k}\right) \\
& =\frac{1}{\left|A_{k}\right|^{2}+\left|B_{k}\right|^{2}}\left(1+V_{k} \cos \left(\Delta \varphi_{k}\right)\right)
\end{aligned}
$$

где $V_{k}=2\left|A_{k}\right|\left|B_{k}\right| / \sqrt{\left|A_{k}\right|^{2}+\left|B_{k}\right|^{2}}-$ интерференционный контраст.

На рис. $2, b$ показаны фазовые составляющие $\Delta \varphi_{k}$, сформированные в оптических сигналах в плоскости каждого фотоприемника после прохождения всей оптической схемы. Пары ФП1 и ФП2, а также ФПЗ и ФП4 можно использовать для определения величины линейного перемещения $x$ при условии сохранения разности фаз $90^{\circ}$ между итоговыми оптическими сигналами на фотоприемниках [9]. К такой реализации приводит комбинация фазовых сдвигов $\Phi_{1}, \Phi_{2}$ и $\Phi_{3}$, сформированных в оптических сигналах после прохождения излучения через АШ в оптической схеме.

Таким образом, АШ в схеме должна не только обеспечивать эффективное деление пучка, но и начальную разность фаз для квадратурной модуляции пар интерференционных сигналов. В условиях рассматриваемой задачи моделирования одинаково важно учитывать как накопленную фазовую составляющую, так и энергетический расчет для каждого из пучков. 


\section{Дифракционная решетка как элемент внесения фазового сдвига}

Вносимые в дифрагировавшие пучки фазовые сдвиги (как показано на рис. $2, a, b$ ) в представленном моделировании рассматриваются для пучка 0 -го порядка относительно \pm 1 -х порядков и представляют собой разности накопленных фаз [10]. В частности, $\Phi_{1}-$ фазовый сдвиг, полученный при нормальном падении излучения со стороны АШ в процессе первой дифракции, $\Phi_{2}$ - аналогичный сдвиг фазы, но при освещении со стороны КШ при второй дифракции на АШ, $\Phi_{3}-$ фазовый сдвиг, который обеспечивается при наклонном падении излучения со стороны решетки. Неравенство представленных значений для различных условий освещения подтверждено экспериментально и при решении задачи дифракции на основе электромагнитной теории [11]. Если бы все три случая обеспечивали одинаковый фазовый сдвиг $\Phi$, то это упростило бы формирование фазовой составляющей $\varphi_{k}$ для боковых приемников излучения ФП1 и $Ф П 4$. Она бы включала удвоенный фазовый сдвиг $2 \Phi$, а на осевые фотоприемники $Ф П 2$ и $Ф П 3$ в таком случае поступали бы интерференционные сигналы только с вкладом $\Omega(x)$. Такой подход не является физически справедливым и на практике приводит к значительным отклонениям параметров модуляции итоговых сигналов от ожидаемых. Для конкретной схемы оптического датчика линейных перемещений, если излучение дифрагирует несколько раз, каждый случай требует собственного анализа.

Накапливаемые фазы в каждом из дифракционных порядков физически зависят от пространственных параметров анализирующей шкалы (высота, период, фактор заполнения, геометрическая форма профиля пропускающей дифракционной решетки) и изменяются при различных условиях освещения (длина волны, поляризация, угол падения и др.). Строгий подход к решению задачи дифракции математически описывает преобразование электромагнитного поля и позволяет понять процесс аккумуляции фазы в зоне модуляции дифракционной решетки [12]. В математической реализации моды в наборе плоских волн, распространяющихся под разными углами для всех направлений дифракции, складываются на выходе зоны модуляции дифракционной решетки, таким образом формируя значение комплексной амплитуды. Абсолютное значение фазы (как и амплитуды) для каждого дифракционного порядка содержит вклад каждой моды, существующей в рассматриваемом направлении. В свою очередь фаза для каждой моды зависит от входных параметров задачи дифракции: длины волны света, условия поляризации, углов падения и азимута, показателей преломления материалов и пространственных характеристик геометрического профиля.

\section{Моделирование фазового распределения в оптической схеме интерференционного датчика линейных перемещений}

Чтобы сформулировать требования к АШ, в качестве которой используется пропускающая дифракционная решетка, необходимо проанализировать совместное влияние фаз, накапливающихся по ходу распространения каждого светового пучка от источника излучения до плоскости интерференции. Для конкретной схемы датчика, если свет дифрагирует несколько раз, как в нашем случае, каждый случай требует собственного расчета. Расчет оптической схемы интерференционного датчика линейных перемещений, приведенной на рис. 1, сводится к расчету фазовых сдвигов, вносимых в соответствующие дифракционных порядки решеток с помощью электромагнитной теории дифракции $[12,13]$. В частности, необходимо рассчитать результирующую фазу света для пары оптических сигналов при линейном перемещении и значения интерференционного контраста, сформированного на каждом фотоприемнике

Численное решение задачи дифракции было проведено в среде Matlab. Для моделирования дифракции на решетке с прямоугольным профилем использован строгий метод RCWA (rigorous coupled-wave analysis) [13]. Для моделирования синусоидальных решеток использовано аналогичное программное обеспечение с реализованным методом криволинейных координат, который применяется для анализа структур со сглаженным профилем рельефа [14].

Далее приведено описание исходных данных для расчета. Рассмотрено два случая скрещенных поляризаций (ТЕ и ТМ). Длина волны падающего излучения от полупроводникового лазера соответствует красному спектру и составляет $660 \mathrm{~nm}$. Зона модуляции дифракционной решетки выполнена из полиметилметакрилата с диэлектрической проницаемостью $\varepsilon_{\mathrm{sub}}=2.21$. Положка дифракционной решетки выполнена из стекла К8 с диэлектрической проницаемостью $\varepsilon_{\mathrm{gr}}=2.29$. Абсолютное значение фазы, накопленной в подложке дифракционной решетки, не влияет на результат вычисления разности фаз в силу симметрии оптической схемы. При моделировании были выбраны типовые значения параметров дифракционной решетки, используемых на практике, а именно периоды АШ в диапазоне от 0.8 до $1.8 \mu \mathrm{m}$ высоты профиля рельефа в диапазоне от нулевого значения до $1.8 \mu \mathrm{m}$. Рассматриваются синусоидальный рельеф и прямоугольный рельеф с различным фактором заполнения структуры (отношением ширины выступа к периоду).

Требования для выбора конкретных параметров рельефа пропускающей дифракционной решетки сформированы для удовлетворения задачи квадратурной модуляции.

В электронном блоке электрические сигналы, полученные с фотоприемников, оцифровываются внутри 


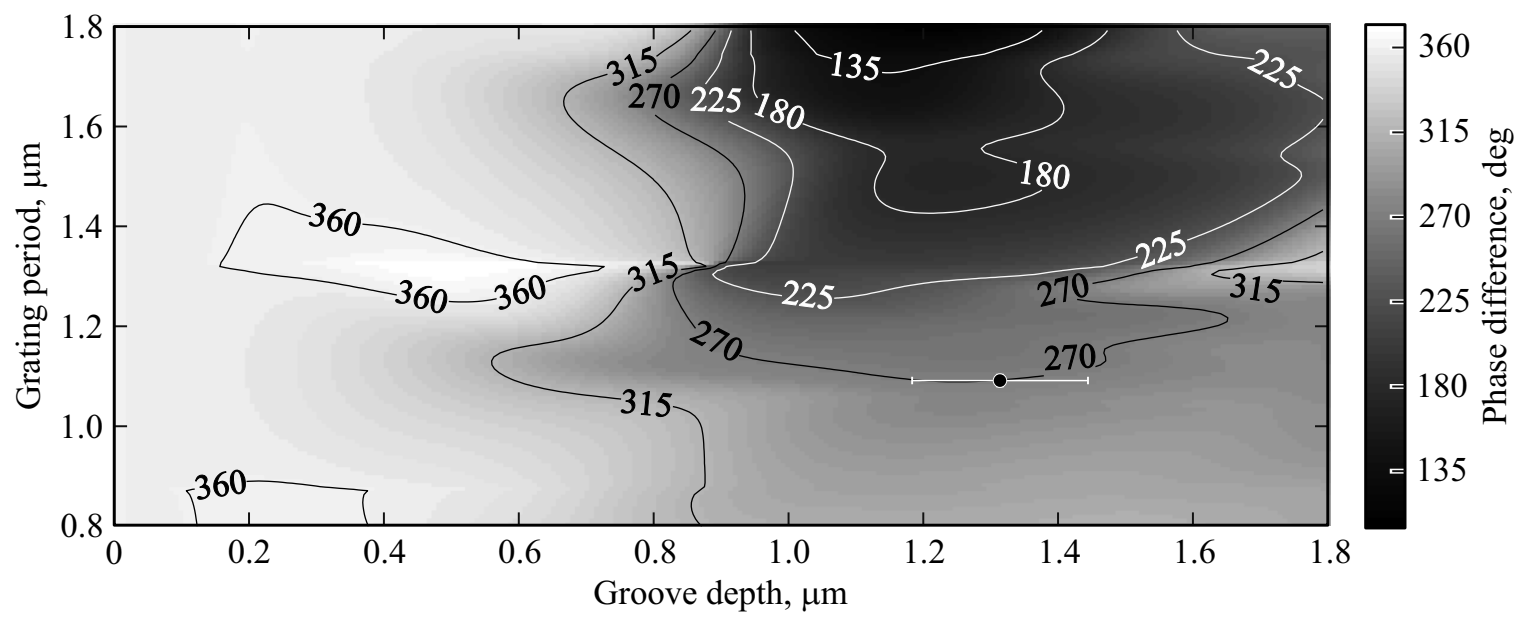

Рис. 3. Контурные диаграммы одинаковой разности фаз для синусоидального профиля дифракционной решетки (АШ), случай ТЕ-поляризации.

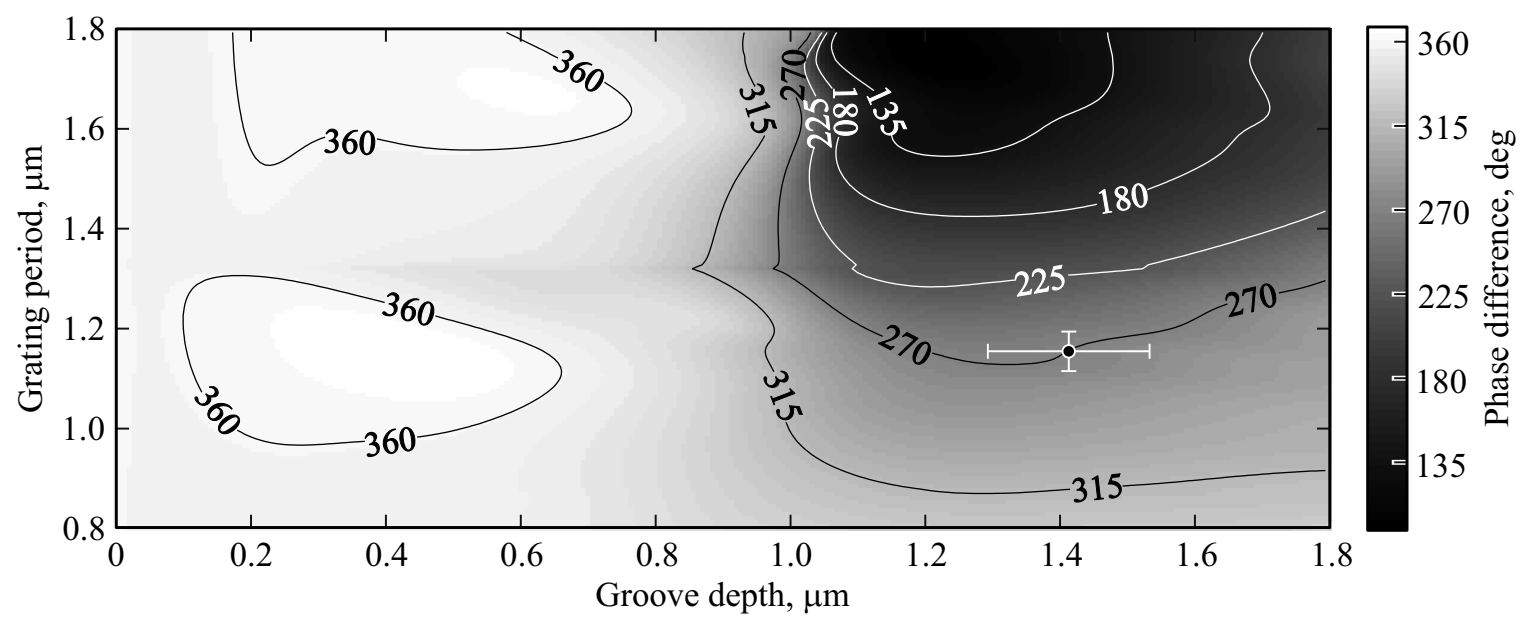

Рис. 4. Контурные диаграммы одинаковой разности фаз для синусоидального профиля дифракционной решетки (АШ), случай ТМ-поляризации.

периода на требуемое количество отсчетов. К примеру, если период кодирующей шкалы составляет $d=1 \mu \mathrm{m}$, то интерференционный период оптического сигнала, поступающего на фотоприемник, составляет $d / 2=500 \mathrm{~nm}$ в силу присутствия удвоенной фазовой составляющей $\Omega$ (рис. $2, b)$. Тогда, если мы хотим добиться погрешности определения перемещения $\pm 0.5 \mathrm{~nm}$, нам необходимо провести 1000-кратную интерполяцию. Таким образом, на основе требований высокого разрешения отклонение расчетной фазы от значения, кратного $90^{\circ}$, не должно превышать $\pm 0.36^{\circ}$ или $\pm 2 \pi / 1000 \mathrm{rad}$, что соответствует тысячной доле периода. Значение интерференционного контраста $V$ оптических сигналов, поступающих на каждый фотоприемник ниже, чем 0.85. Данное значение выбрано для обеспечения глубины модуляции как оптического, так и последующего электрического сигнала. Для анализирующей шкалы также установлены требования по дифракционной эффективности (ДЭ): не ниже $20 \%$, как в 0 -м, так и в \pm 1 -х порядках. На практике для тонких рельефно-фазовых решеток, применяемых в качестве измерительных шкал [15], дифракционная эффективность составляет 20-25\%, что ниже теоретического предела [16].

На рис. 3, 4 приведены контурные диаграммы одинаковой фазы в виде зависимости разности фаз оптических сигналов перемещения $\Delta \Psi_{1-2}=\Delta \varphi_{1}-\Delta \varphi_{2}=\Phi_{3}+\Phi_{2}$ между ФП1 и ФП2 (рис. 2,b) от периода и глубины рельефа пропускающей дифракционной решетки с синусоидальным профилем. Значение разности фаз оптических сигналов перемещения $\Delta \Psi_{3-4}$ между ФП3 и ФП4 по модулю равно $\Delta \Psi_{1-2}$ (но противоположно по знаку), поэтому отдельно не приводится (рис. $2, b$ ). Разность фаз $\Delta \Psi_{3-4}$ между ФП3 и ФП4 всегда составляет $180^{\circ}$ из-за симметричности оптической схеме (рис. 2, $b$ ). Рисунки 3 и 4 соответствуют ТЕ- и ТМ-поляризациям. Значение разности фаз приведено к диапазону от 0 до $360^{\circ}$. Контурами показаны линии разности фаз с шагом $45^{\circ}$. Моделирование показало, что синусоидальный профиль 


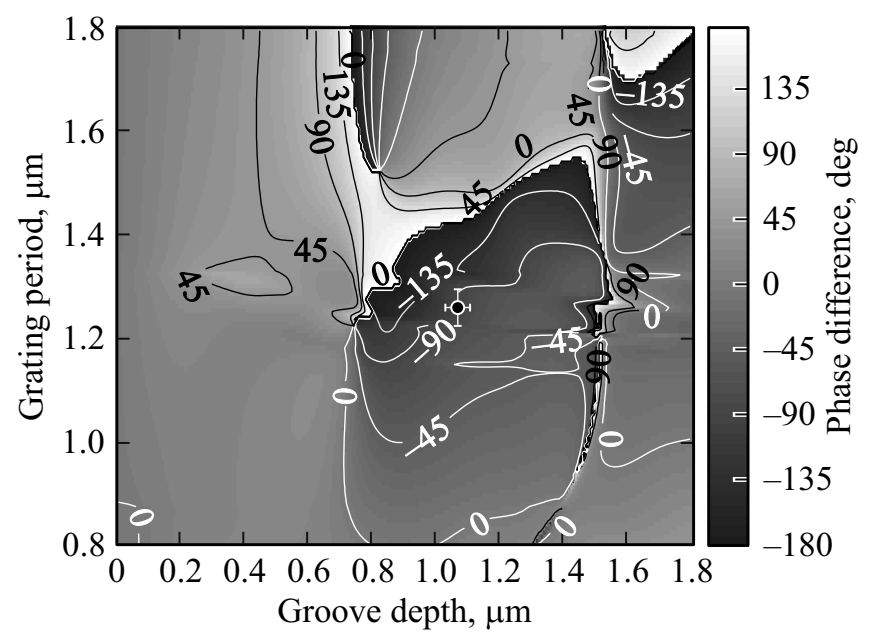

Рис. 5. Контурные диаграммы одинаковой разности фаз для прямоугольного профиля с фактором заполнения 0.3 , случай ТЕ-поляризации.

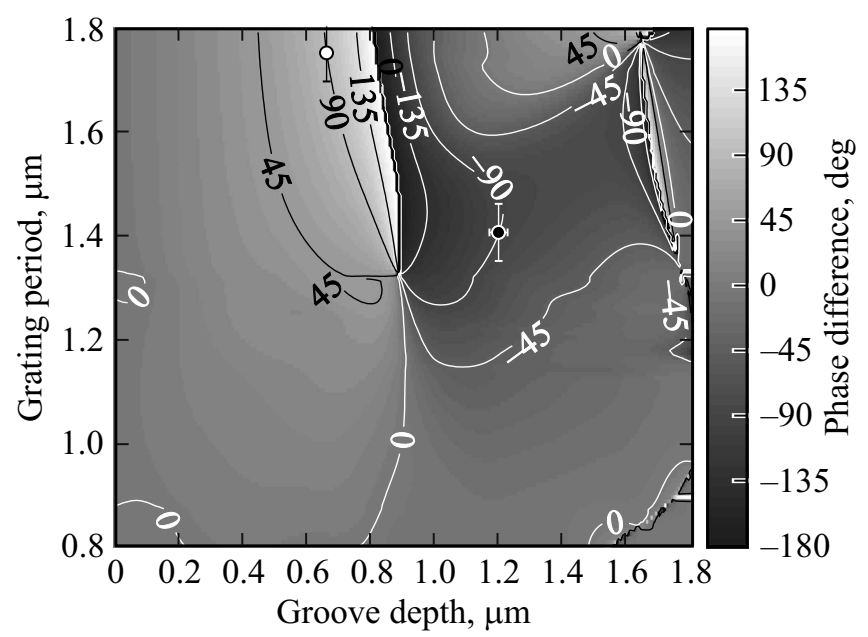

Рис. 6. Контурные диаграммы одинаковой разности фаз для прямоугольного профиля с фактором заполнения 0.3 , случай ТМ-поляризации.

с глубиной менее $800 \mathrm{~nm}$ не обеспечивает какого-либо фазового сдвига между результирующими оптическими сигналами (соответственно итоговыми электрическими сигналами). Белые окружности с отрезками или крестами на рис. 3,4 и далее показывают решения, обеспечивающие описанные выше требования по квадратурной модуляции оптических сигналов и энергетические требования к ним.

Для ТЕ-поляризации (рис. 3) решение предоставляет более широкий допуск на высоту профиля рельефа, но малую возможность к варьированию периода. Решение для ТМ-поляризации (рис. 4) соответствует достаточно большому допуску как для высоты профиля, так и для периода.

На рис. 5-8 приведены аналогичные контурные диаграммы одинаковой фазы в виде зависимости разности фаз $\Delta \Psi_{1-2}$ оптических сигналов между фотоприемниками ФП1 и ФП2 от периода и глубины профиля уже для прямоугольного рельефа пропускающей дифракционной решетки (АШ). Рисунки 5 и 6 соответствуют случаям TЕ- и ТМ-поляризации для прямоугольного рельефа АШ с фактором заполнения 0.3. Рисунки 7 и 8 иллюстрируют соответственно случаи ТЕ- и ТМ-поляризации для прямоугольного рельефа АШ с фактором заполнения 0.5. Значение разности фаз приведено к диапазону от -180 до $180^{\circ}$, а контурами показаны линии разности фаз с шагом $45^{\circ}$.

В таблице приведены геометрические параметры АШ и соответствующие энергетические параметры оптической схемы, которые позволяют обеспечить разность фаз, кратную $90 \pm 0.36^{\circ}$. Некоторое сочетание периода и высоты рельефа шкал позволяет выполнить данное условие для каждого из приведенных типов профиля

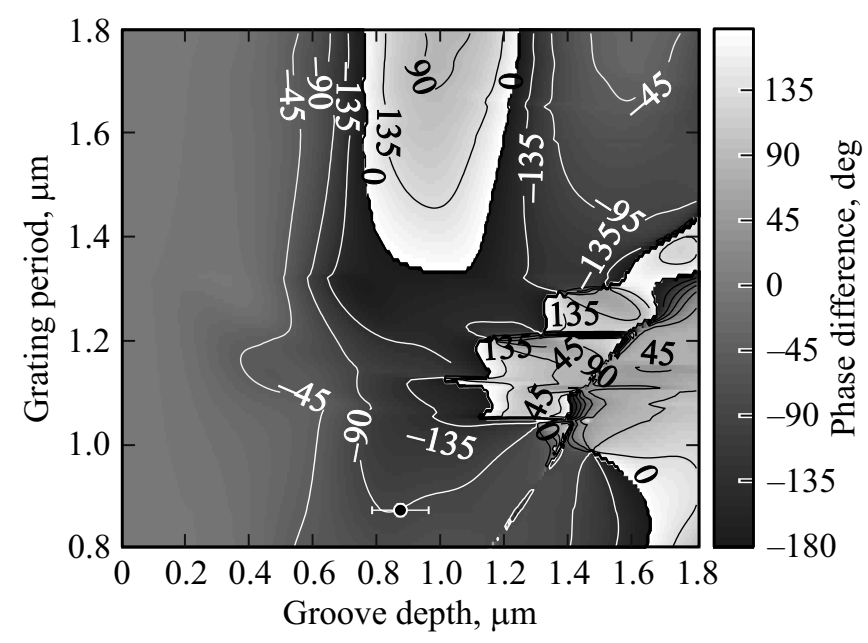

Рис. 7. Контурные диаграммы одинаковой разности фаз для прямоугольного профиля с фактором заполнения 0.5 , случай ТЕ-поляризации.

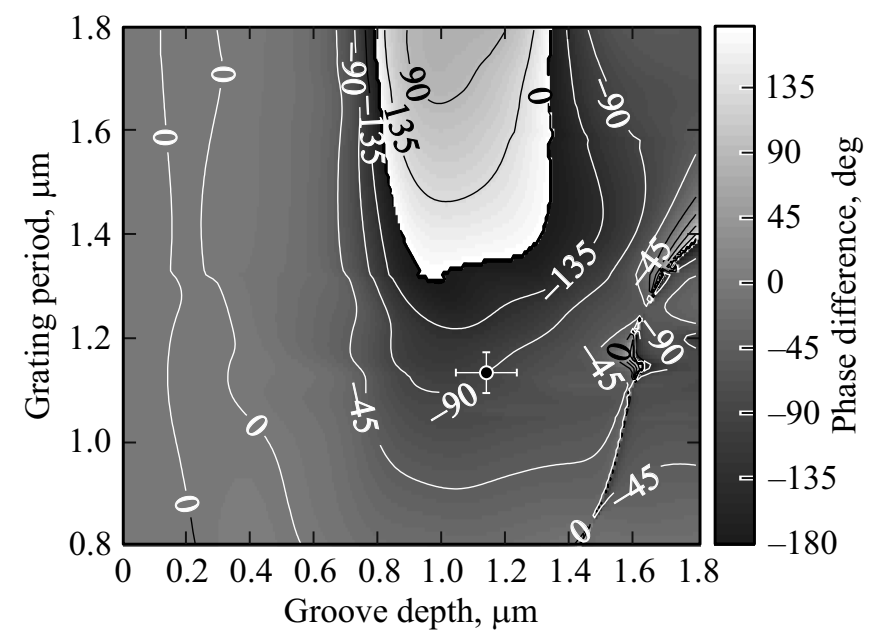

Рис. 8. Контурные диаграммы одинаковой разности фаз для прямоугольного профиля с фактором заполнения 0.5 , случай ТМ-поляризации. 
Рассчитанные параметры анализирующей шкалы для формирования квадратурных сигналов

\begin{tabular}{|c|c|c|c|c|c|c|c|c|}
\hline $\begin{array}{c}\text { Тип профиля } \\
\text { рельефа }\end{array}$ & $\begin{array}{c}\text { Состояние } \\
\text { поляризации }\end{array}$ & $\begin{array}{c}\text { Разность фаз } \Delta \Psi_{1-2} \\
\left(\text { или }-\Delta \Psi_{3-4}\right), \mathrm{deg}\end{array}$ & $\begin{array}{c}\text { ДЭ для } \\
\text { ФП1 }\end{array}$ & $\begin{array}{c}\text { ДЭ для } \\
\text { ФП2 }\end{array}$ & $\begin{array}{c}\text { Контраст } \\
\text { на ФП1 }\end{array}$ & $\begin{array}{c}\text { Контраст } \\
\text { на } Ф П 2\end{array}$ & $\begin{array}{c}\text { Период, } \\
\text { nm }\end{array}$ & $\begin{array}{c}\text { Высота } \\
\text { профиля, } \mathrm{nm}\end{array}$ \\
\hline \multirow{2}{*}{ Синусоидальный } & $\mathrm{TE}$ & \multirow{2}{*}{$270 \pm 0.36$} & 0.099 & 0.176 & 0.952 & 1.000 & $1091 \pm 12$ & $1314 \pm 130$ \\
\hline & $\mathrm{TM}$ & & 0.121 & 0.185 & 0.921 & 1.000 & $1154 \pm 40$ & $1412 \pm 120$ \\
\hline \multirow{3}{*}{$\begin{array}{c}\text { Прямоугольный } \\
\text { с фактором } \\
\text { заполнения } 0.3\end{array}$} & $\mathrm{TE}$ & \multirow{2}{*}{$-90 \pm 0.36$} & 0.122 & 0.184 & 0.984 & 1.000 & $1257 \pm 35$ & $1067 \pm 38$ \\
\hline & $\mathrm{TM}$ & & 0.123 & 0.173 & 0.911 & 1.000 & $1406 \pm 55$ & $1194 \pm 28$ \\
\hline & $\mathrm{TM}$ & $90 \pm 0.36$ & 0.118 & 0.087 & 0.712 & 1.000 & $1753 \pm 55$ & $658 \pm 14$ \\
\hline \multirow{2}{*}{$\begin{array}{c}\text { Прямоугольный } \\
\text { с фактором } \\
\text { заполнения } 0.5\end{array}$} & TE & \multirow{2}{*}{$-90 \pm 0.36$} & 0.119 & 0.229 & 0.999 & 1.000 & $871 \pm 12$ & $870 \pm 88$ \\
\hline & TM & & 0.113 & 0.211 & 0.977 & 1.000 & $1131 \pm 39$ & $1138 \pm 95$ \\
\hline
\end{tabular}

(синусоидального, прямоугольного с факторами заполнения 0.3 и 0.5 ) и получить стабильные оптические сигналы. Изменение геометрической формы профиля позволяет добиться технологически более простого в реализации сочетания периода и высоты профиля рельефа [17]. Для энергетической оценки приведены значения интерференционного контраста, а также суммарная дифракционная эффективность (ДЭ) для оптических сигналов, поступивших на приемники ФП1 и ФП2 (рис. 1).

Моделирование показало необходимость изготовления относительно .глубокого. рельефа по высоте профиля, превышающего значение периода. Глубина профиля может превышать ширину выступа в три раза, что технологически вполне реализуемо [18], хоть и может повлечь некоторые технологические сложности при изготовлении. Для прямоугольного профиля рельефа с фактором заполнения 0.3 отдельно приведены параметры для реализации квадратурной модуляции оптических сигналов, когда высота профиля значительно меньше периода. В этом случае наблюдается падение интерференционного контраста на боковом фотоприемнике и уменьшение эффективности потока излучения, приходящего на осевой фотоприемник.

Необходимо отметить, что приведенные отклонения для периода и высоты профиля, представленные в таблице, позволяют обеспечить требуемое значение разности фаз, а не являются технологическими допусками на изготовление. При создании датчиков линейных перемещений важную роль играет постоянство значения периода в пределах всей рабочей зоны шкал. От значения периода напрямую зависит период гармонических квадратурных сигналов перемещения, а следовательно, и разрешение датчика. Непостоянство периода может привести к нарушению геометрии хода лучей и соответствующему смещению интерференционной полосы в плоскости фотоприемников. Для конкретного датчика линейных перемещений после выбора величины периода в соответствии с расчетами устанавливается допуск на изменение глубины профиля рельефа.
Сдвиг фазы, приобретаемый при каждой дифракции в схеме, чувствителен к поляризации. Для подобранных параметров анализирующей шкалы одно и то же значение разности фаз для скрещенных поляризаций обеспечивается при различии глубины профиля рельефа более чем на 100-200 nm. Аналогичный вывод справедлив и для значений периода. Например, для прямоугольного профиля с фактором заполнения 0.3 рассчитанные высоты для разных поляризаций различаются на $127 \mathrm{~nm}$, а периоды на $149 \mathrm{~nm}$. Выбор параметров дифракционной решетки, функционирующей как устройство внесения фазового сдвига, возможен и независимо от поляризации. Но подобное решение будет сопровождаться как снижением дифракционной эффективности, так и отклонением вносимого фазового сдвига. На практике мы рекомендуем использовать ТЕ-поляризацию. В таком случае вектор напряженности электрического поля колеблется согласно направлениям штрихов дифракционной решетки и не претерпевает поворота. Использование ТМ-поляризации требует дополнительного исследования поляризационных свойств анализирующей шкалы [19].

\section{Заключение}

Проектирование высокоточных интерференционных датчиков линейных перемещений требует проведения как анализа фазовых соотношений для каждого из интерферирующих пучков, так и энергетического расчета оптической системы. Применение в качестве анализирующей шкалы дифракционной решетки, изготовленной только в соответствии с требованиями деления пучка, влечет за собой формирование случайного соотношения фаз. Это, в свою очередь, может привести к невозможности создания квадратурной модуляции оптических сигналов. Представленный в работе анализ позволяет реализовать стабильную квадратурную разность фаз в схеме интерференционного датчика линейных перемещений, не зависящую от сложной юстировки оптической схемы. Результаты расчета показывают, что для создания 
разности фаз с высокой стабильностью можно использовать достаточно широкий допуск на высоту профиля рельефа.

Рельефно-фазовые дифракционные решетки обеспечивают внесение фазового сдвига в одном направлении дифракции относительно другого. Представлены параметры анализирующей шкалы и соответствующие энергетические параметры оптической схемы, которые позволяют обеспечить стабильную разность фаз, кратную $90^{\circ}$, с погрешностью не более $\pm 0.36^{\circ}$, что достаточно для обеспечения работоспособности датчика с нанометрическим разрешением. Приводятся данные по соотношению периода и высоты для каждого из приведенных типов синусоидального и прямоугольного профилей с факторами заполнения 0.3 и 0.5 анализирующей дифракционной решетки.

\section{Финансирование работы}

Работа выполнена при поддержке Российского научного фонда (Проект № 18-79-00304).

\section{Конфликт интересов}

Авторы заявляют, что у них нет конфликта интересов.

\section{Список литературы}

[1] Lv Q., Liu Z., Wang W., Li X., Li S., Song Y., Yu H., Li W. // Appl. Opt. 2018. V. 57. N 31. P. 9455. doi 10.1364/AO.57.009455

[2] Hausotte T., Percle B., Gerhardt U., Dontsov D., Manske E., Jager G. // Meas. Sci. Technol. 2012. V. 23. P. 074004. doi 10.1088/0957-0233/23/7/074004

[3] Lee J.-Y., Jiang G.-A. // Opt. Exp. 2013. V. 21. N 21. P. 25553. doi 10.1364/OE.21.025553

[4] Комоцкий В.А., Корольков В.И., Соколов Ю.М. // Автометрия. 2006. Т. 42. № 6. С. 105-112.

[5] Jourlin Y., Jay J., Parriaux O. // Precis. Engineering. 2002. V. 26. P. $1-6$.

[6] Ye G., Fan S., Liu H., Li X., Yu H., Shi Y., Yin L., Lu B. // Meas. Sci. Technol. 2014. Vol. 25. P. 125003. doi 10.1088/0957-0233/25/12/125003

[7] Yaqoob Z., Wu J., Cui X., Heng X., Yang C. // Opt. Express. 2006. V. 14. P. 8127.

[8] Teimel A. // Precis. Engineering. 1992. V. 4. P. 147154.

[9] Шишова М.В., Одиноков С.Б., Лушников Д.С., Жердев А.Ю., Гурылев О.А. // III Международная конференция и молодежная школа „Информационные технологии и нанотехнологии“: Сб. трудов. Самара, 2017. С. 1438.

[10] Вишняков Г.Н., Левин Г.Г., Минаев В.Л. // Опт. и спектр. 2015. T. 118. № 6. C. 1005. doi 10.7868/S0030403415060240

[11] Cordeiro C.M.B., Cescato L., Freschi A.A., Lifeng Li // Opt. Lett. 2003. V. 28. N 9. P. 683.

[12] Clausnitzer T., Kämpfe T., Kley E.-B., Tünnermann A., Tishchenko A.V., Parriaux O. // Opt. Express. 2008. V. 16. N 8. P. 5577. doi 10.1364/OE.16.005577

[13] Moharam M.G., Grann E.B., Pommet D.A., Gaylord T.K. // J. Opt. Soc. Am. A. 1995. V. 12. N 5. P. 1068.
[14] Chandezon J., Maystre D., Raoult G. // J. Optics (Paris). 1980. V. 11. P. 235.

[15] Шишова М.В., Одиноков С.Б., Лушников С.Д., Жердев А.Ю. // Опт. журнал. 2018. Т. 85. № 7. doi 10.17586/1023-5086-2018-85-07-27-32

[16] Одиноков С.Б. и др. // Вестник МГТУ. Сер. Приборостроение. 2005. Т. 61. № 4. С. 8.

[17] Аксенов В.П., Венедиктов В.Ю., Севрюгин А.А., Турсунов И.М. // Опт. и спектр. 2018. Т. 124. № 2. C. 275; Aksenov V.P., Venediktov V.Y., Sevryugin A.A., Tursunov I.M. // Opt. Spectrosc. 2018. V. 124. N 2. P. 273.

[18] Zheng X., Wang Q., Zhang R., Ma L., Luan J. // Scientific Reports. 2018. V. 8. P. 12720. doi 10.1038/s41598-018-31194-y

[19] Azzam R.M.A., Bashara N.M. // Phys. Rev. B. 1972. V. 5. N 12. P. 4721. 